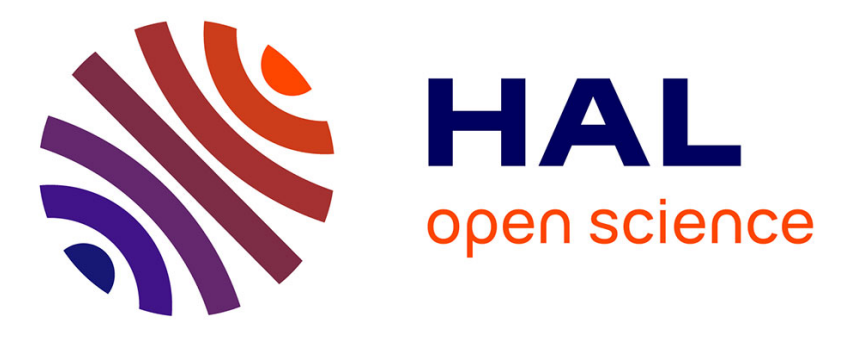

\title{
Importance of Vacancies and Doping in the Hole-Transporting Nickel Oxide Interface with Halide Perovskites
}

Boubacar Traoré, Laurent Pedesseau, Jean-Christophe Blancon, Sergei Tretiak, Aditya D Mohite, Jacky Even, Claudine Katan, Mikael Kepenekian

\section{To cite this version:}

Boubacar Traoré, Laurent Pedesseau, Jean-Christophe Blancon, Sergei Tretiak, Aditya D Mohite, et al.. Importance of Vacancies and Doping in the Hole-Transporting Nickel Oxide Interface with Halide Perovskites. ACS Applied Materials \& Interfaces, 2020, 12 (5), pp.6633-6640. 10.1021/acsami.9b19457 . hal-02472160

\section{HAL Id: hal-02472160 \\ https://hal-univ-rennes1.archives-ouvertes.fr/hal-02472160}

Submitted on 30 Mar 2020

HAL is a multi-disciplinary open access archive for the deposit and dissemination of scientific research documents, whether they are published or not. The documents may come from teaching and research institutions in France or abroad, or from public or private research centers.
L'archive ouverte pluridisciplinaire HAL, est destinée au dépôt et à la diffusion de documents scientifiques de niveau recherche, publiés ou non, émanant des établissements d'enseignement et de recherche français ou étrangers, des laboratoires publics ou privés. 


\title{
Importance of vacancies and doping in hole
} transporting nickel oxide interface with halide perovskites

\author{
Boubacar Traore, ${ }^{* \dagger} \dagger$ Laurent Pedesseau, ${ }^{\dagger}$ Jean-Christophe Blancon, ${ }^{\complement}$ Sergei \\ Tretiak, ${ }^{\S}$ Aditya D. Mohite, ${ }^{\circledR}$ Jacky Even, ${ }^{\dagger}$ Claudine Katan, ${ }^{\ddagger}$ and Mikaël \\ Kepenekian*,‡ \\ †Univ Rennes, INSA Rennes, CNRS, Institut FOTON - UMR 6082, F-35000 Rennes, \\ France \\ $\ddagger$ Univ Rennes, ENSCR, CNRS, ISCR - UMR 6226, F-35000 Rennes, France \\ \Department of Chemical and Biomolecular Engineering, Rice University, Houston, TX \\ 77005. \\ $\S$ Los Alamos National Laboratory, Los Alamos, NM 87545, U.S.A. \\ E-mail: boubacar.traore@insa-rennes.fr; mikael.kepenekian@univ-rennes1.fr
}




\begin{abstract}
Nickel oxide $(\mathrm{NiO})$ is a commonly used contact material for a variety of thin-film optoelectronic technologies based on organic or hybrid materials. In such setups, interfaces play a crucial role as they can reduce, if not kill, the device performances by bringing additional traps or energy barriers hindering charge carriers extraction from the active layer. Here, we computationally examine a prototype halide perovskite architecture, $\mathrm{NiO} / \mathrm{MAPbI}_{3}\left(\mathrm{MA}=\mathrm{CH}_{3} \mathrm{NH}_{3}^{+}\right)$, which has shown excellent photovoltaic performance and in particular a large open-circuit voltage. We show that efficient hole collection is only achieved when considering the role of vacancies induced by standard material deposition techniques. Specifically, Ni vacancies lead to nearly perfect valence band energy level alignment between the active layer and the contact material. Finally, we show how Li doping greatly improves the performances of the device and further propose alternative dopants. Our results suggest the high tunability of $\mathrm{NiO}$ interfaces for the design of optimized optoelectronic devices far beyond halide perovskites.
\end{abstract}




\section{Introduction}

Tuning interface energetics in thin film optoelectronic devices is of paramount importance. In such devices, the photo-active material is typically sandwiched between charge collection layers, which are referred to as electron transport layer (ETL) and hole transport layer (HTL). Poorly matched and designed interfaces can lead to energy level and dielectric mismatches along with traps causing undesired charge carriers recombination that may overall reduce the performances of the photo-active layer. ${ }^{1,2}$ Hence, their selection and optimization play an essential role in the performance of optoelectronic devices. ${ }^{2-4}$ Among HTL materials, the inorganic transition metal-oxide nickel oxide $(\mathrm{NiO})$, is an attractive technological and relatively inexpensive solution including low-cost solution process deposition techniques. ${ }^{5}$ Its adoption in photovoltaics was triggered by the pioneering works of He et al. ${ }^{6}$ and Irwin et $a l .{ }^{7}$ in dye-sensitized solar cells (DSSC) and organic photovoltaic (OPV) devices, respectively. Moreover, $\mathrm{NiO}$ finds extensive applications as a transparent conductive oxide, ${ }^{8}$ in water splitting ${ }^{9}$ and batteries. ${ }^{10}$ The interest for $\mathrm{NiO}$ in these diversified fields takes root from its wide band gap $(>3.6 \mathrm{eV}), p$-type conduction and magnetic and optical refraction properties.

Recently, some of those features were exploited in the context of hybrid organic-inorganic perovskites (HOP) based optoelectronic devices. These studies have been motivated by the power conversion efficiency of HOP-based photovoltaics, which nowadays competes with that of silicon solar cells. ${ }^{11}$ In particular, HOP solar cells exhibit attractive photovoltaic performances when $\mathrm{NiO}$ is used as a HTL in stable large-area architectures or monolithic $\mathrm{HOP} /$ silicon tandem devices. ${ }^{12-16}$ The improved performance was attributed to the near ideal valence band energy alignment between $\mathrm{NiO}$ and, for instance, methylammonium (MA $=\mathrm{CH}_{3} \mathrm{NH}_{3}^{+}$) lead iodide $\mathrm{MAPbI}_{3} \cdot{ }^{17,18}$ Besides, the experimental measurements point to an increased photovoltaic power conversion efficiency when $\mathrm{NiO}$ is doped with $\mathrm{Li}^{18}$ Few theoretical works describe the interface of halide perovskites with $\mathrm{TiO}_{2},{ }^{19}$ even fewer deal with other HTL. ${ }^{20}$ As a consequence, the fundamental understanding of the origin of such a 
near ideal alignment between $\mathrm{NiO}$ and $\mathrm{MAPbI}_{3}$ with and without $\mathrm{Li}$ doping is lacking.

Here, we study an archetypal interface through first-principle calculations based on density functional theory (DFT) by examining in detail NiO junctions with the prototypical HOP material, $\mathrm{MAPbI}_{3}$. Considering the defect-free interface, we find that the high-frequency dielectric constant profile across the interface presents a low dielectric contrast between the two materials, which benefits hole extraction. However, our results show that the valence band energy level alignment between pristine $\mathrm{NiO}$ and $\mathrm{MAPbI}_{3}$ materials is not favourable for hole extraction. In contrast, we show a significant change in the valence band energetics of $\mathrm{NiO}$ due to native $\mathrm{Ni}$ vacancies appearing in the material deposition. This is consistent with ultraviolet photoelectron spectroscopy (UPS) experiments. Therefore, we demonstrate that the widely accepted view of near ideal experimental valence band energy level alignment between $\mathrm{NiO}$ and $\mathrm{MAPbI}_{3}$ is related to these $\mathrm{Ni}$ vacancies. Finally, we show that Li doping improves the level alignment and propose $\mathrm{Cs}$ and $\mathrm{Rb}$ as alternative dopants of $\mathrm{NiO}$ to further tune its interfacial energetics. The methodology developed in this report will be directly transferable to the understanding of other types of interfaces including various perovskites, conventional semiconductors, and alternative carrier transport layer materials.

\section{Results and discussion}

$\mathrm{NiO}$ and $\mathrm{MAPbI}_{3}$ bulk materials $\mathrm{NiO}$ is a prototype transition metal oxide that has been extensively scrutinized over the years. ${ }^{21-24}$ The localized Ni 3d electrons make it a highly correlated system, whose theoretical description fails in the local density approximation (LDA) or the generalized gradient approximation (GGA) of DFT. ${ }^{25}$ Hence, we adopted the so-called $\mathrm{LDA}+\mathrm{U}($ or GGA $+\mathrm{U}) \operatorname{method}^{26,27}$ by applying a Hubbard-like correction term $\mathrm{U}$ to Ni 3d orbitals within the SIESTA code (see Supporting Information, SI). ${ }^{28,29}$ In previous works, the method has been successful in describing the magnetic properties of $\mathrm{NiO}$ and results in a band gap that is in a fair agreement with experiment. ${ }^{26,27}$ In our case, we found 
that an effective $\mathrm{U}$ value $\left(\mathrm{U}_{\text {eff }}\right)$ of $6.0 \mathrm{eV}$ was well suited to capture the main features of the electronic structure of $\mathrm{NiO}$ and is consistently used across the different calculations for comparison. We note that this value falls within the accepted range of appropriate $U$ values reported for $\mathrm{NiO}$ in the literature. ${ }^{27,30}$ The detailed description of the electronic structure of $\mathrm{NiO}$ and the selection of $\mathrm{U}_{\text {eff }}$ can be found in the SI (Text S1). Briefly, the electronic band gap of $\mathrm{NiO}$ corresponds to a charge transfer type excitation (Text S2, SI) and the calculated magnetic moment of $\mathrm{Ni}\left(1.77 \mu_{B}\right)$ is in excellent agreement with the experimental value $\left(\sim 1.7 \mu_{B}\right) .{ }^{31}$ Moreover, the computed relaxed lattice constant $(\mathrm{a}=4.25 \AA)$ presents less than $2 \%$ error as compared to the experimental structure $(\mathrm{a}=4.167 \AA){ }^{32}$

The $\mathrm{MAPbI}_{3}$ perovskite and its alloys, involving formamidinium cations and mixed cations/halides, have so far produced the most efficient solar cells. ${ }^{33,34}$ Structurally, $\mathrm{MAPbI}_{3}$ forms a 3-dimensional lattice where corner-shared $\mathrm{PbI}_{6}^{2-}$ octahedra extend in all directions with MA cation tumbling around the center of the cubo-octahedra. Over the past years, impressive chemical and materials engineering of HOP have been carried out not only to increase their efficiencies but also to improve their stabilities and photophysical properties. ${ }^{2,16}$ Here, we use its orthorhombic phase with well-defined positions of MA in order to avoid the issue of the dynamical disorder of the latter in the tetragonal and cubic structures. ${ }^{35}$ This low-temperature phase has been extensively studied and DFT-based calculations provide a correct description of the main electronic features, in particular, the direct bandgap at $\Gamma$ point (Figure S2, SI). Let us note that, because of the presence of the heavy atom $\mathrm{Pb}$, the correct treatment of $\mathrm{MAPbI}_{3}$ should include spin-orbit coupling (SOC). However, the impact of SOC on valence band states is negligible (Figure S2, SI), thus it is reasonable to neglect SOC in our DFT calculations focusing on the $\mathrm{NiO} / \mathrm{MAPbI}_{3}$ interface for hole extraction only (spin polarization, however, is included).

$\mathrm{NiO} / \mathrm{MAPbI}_{3}$ pristine interface Considering the experimentally observed (100) surface of $\mathrm{NiO}$ and corresponding lattice parameters, ${ }^{31,32}$ the $\mathrm{MAPbI}_{3}(010)$ surface affords appro- 
priate lattice matching for a $\mathrm{NiO} / \mathrm{MAPbI}_{3}$ interface (Table S3, SI). The resulting lateral lattice mismatch between $\mathrm{MAPbI}_{3}$ and $\mathrm{NiO}$ is less than $3 \%$. Details related to the interface construction can be found in Text S3 (SI).

To combine $\mathrm{NiO}$ and $\mathrm{MAPbI}_{3}$ at the interface, we terminated $\mathrm{MAPbI}_{3}$ by MAI (Figure 1a) as suggested by STM measurements ${ }^{36}$ and reported to be the favourable termination using DFT. ${ }^{37}$ The interface relaxes such that iodine atoms point towards nickel atoms (Figure 1b) with a Ni-I bond length of about $2.87 \AA$, in good agreement with bulk Ni-I bond distance of $2.78 \AA$ in hexagonal $\mathrm{R}-3 \mathrm{~m} \mathrm{NiI} 2 .{ }^{38}$ The resulting band structure (Figure 1c) shows a direct band gap at $\Gamma$ point. The wavefunction plot of the valence band maximum (VBM) at $\Gamma$ point reveals an anti-bonding hybridization between $\mathrm{Pb}(6 \mathrm{~s})-\mathrm{I}(5 \mathrm{p})$ states only (Figure $1 \mathrm{~d}$ ), whereas the same band shows $\mathrm{NiO}$ states at the in-plane $\mathrm{M}_{\mathrm{xy}}$ point. Stated differently, hole wavefunctions are localized inside the perovskite at $\Gamma$ point while they are delocalized between both the perovskite and $\mathrm{NiO}$ at $\mathrm{M}_{\mathrm{xy}}$ point. The presence of I states at $\mathrm{M}_{\mathrm{xy}}$ point is consistent with Ni-I bond formation and shows an efficient coupling between $\mathrm{MAPbI}_{3}$ and $\mathrm{NiO}$.

Efficient charge separation in solar cell devices, and more generally semiconductor heterostructures, depends on the dielectric properties of the respective materials. If the paired media have different dielectric constants, charge separation through the interface and the subsequent carrier transport may be improved or frustrated depending on the extent of the dielectric mismatch. ${ }^{1}$ In our $\mathrm{NiO} / \mathrm{MAPbI}_{3}$ interface, we evaluated the dielectric constants by considering the high-frequency component $\left(\epsilon_{\infty}\right)$ along the interface direction using a firstprinciple based approach described elsewhere. ${ }^{39,40}$ Figure 2 shows the profile of $\epsilon_{\infty}$ along the interface through $\mathrm{NiO}$ and $\mathrm{MAPbI}_{3}$ layers. In their central bulk-like regions, both $\mathrm{NiO}$ $\left(\epsilon_{\infty}=5.1\right)$ and $\mathrm{MAPbI}_{3}\left(\epsilon_{\infty}=5.4\right)$ have values that are comparable to those calculated for their bulk experimental structures (Figure S4, SI). The calculated values for $\mathrm{NiO}$ and $\mathrm{MAPbI}_{3}$ are also in a good agreement with the experimentally measured values amounting to 5.7 and 6.5 , respectively. ${ }^{41,42}$ At the interface region, the dielectric constant shows 
b

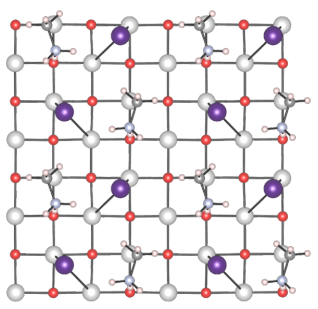

\section{c}

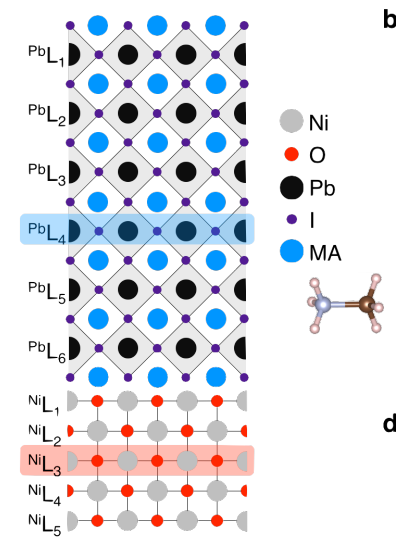

$\psi_{\mathrm{vBM}} @ \Gamma \quad \Psi_{\mathrm{vBM}} @ \mathrm{M}_{\mathrm{xy}}$
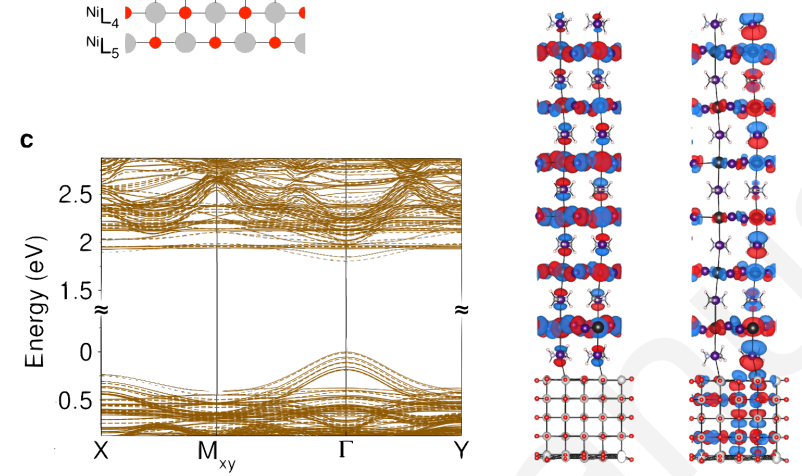

Figure 1: Lattice structure and electronic properties of the $\mathrm{NiO} / \mathrm{MAPbI}_{3}$ interface. a Model used in our calculations and layer labels. b Favorable iodine positions at the interface with I atoms pointing towards $\mathrm{Ni}$ in a bridge-like configuration. Here I, Ni, O, N, C and H atoms are depicted in purple, light gray, red, blue, dark gray and pale pink colors, respectively. c Band structure of the relaxed interface model with a direct bandgap at $\Gamma$ point. The valence band maximum (VBM) at $\Gamma$ point is taken as the energy reference. Plain and dashed lines correspond to majority and minority spins, respectively. d VBM Wavefunction at $\Gamma$ and $\mathrm{M}_{\mathrm{xy}}$ points. Hybridized $\mathrm{MAPbI}_{3}$ and $\mathrm{NiO}$ states appear at $\mathrm{M}_{\mathrm{xy}}$ point while $\mathrm{MAPbI}_{3}$ dominate at $\Gamma$ point.

a local peak, which is, to some extent, related to the $\mathrm{NiO}$ interface states that hybridize with those of $\mathrm{MAPbI}_{3}$, resulting in an interface dipole that we estimated to be about 2.0 Debye. Interestingly, the two dielectric constants $\left(\epsilon_{\infty}\right)$ are comparable indicative of a low dielectric contrast/mismatch between $\mathrm{MAPbI}_{3}$ and $\mathrm{NiO}$. This may hint to an efficient charge separation across the interface due to possible reduced binding energy of holes as a result of low dielectric mismatch. ${ }^{1}$ Noteworthy, in the actual room temperature solar cell operation, low-frequency static dielectric constant $\left(\epsilon_{s}\right)$ also plays an important role and may represent a dominant contribution in the response of charge carriers to the electric field. ${ }^{43}$ Effective $\epsilon_{s}$ values ranging from $11-20$ can be expected in $\mathrm{MAPbI}_{3}$ due to optical phonons and rotational tumbling of organic cations, ${ }^{43,44}$ whereas this value amounts to 11.90 for $\mathrm{NiO} .{ }^{41}$ 


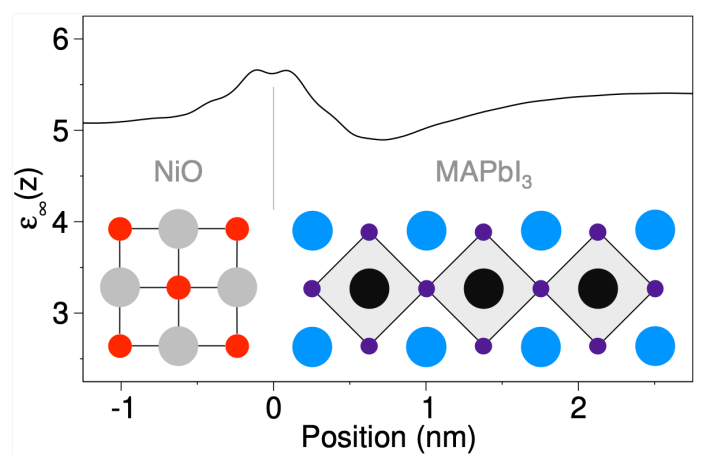

Figure 2: High-frequency dielectric constant profile of the $\mathrm{NiO} / \mathrm{MAPbI}_{3}$ interface. The dielectric mismatch is low, which points to efficient charge extraction at the interface. The inset shows the corresponding position of the $\mathrm{NiO} / \mathrm{MAPbI}_{3}$ slab.

For an efficient hole transmission from the perovskite layer to the hole transport material, the VBM of the latter should be above that of the former. A parameter that quantifies the shift between the VBM of the heterostructure materials is referred to as the valence band offset (VBO). Here, it is defined as the difference between $\mathrm{VBM}_{\mathrm{NiO}}$ and $\mathrm{VBM}_{\mathrm{MAPbI}_{3}}$ from their bulk-like layers. ${ }^{45}$ Therefore, positive values for VBO indicate that $\mathrm{NiO}$ states stand higher in energy than the respective $\mathrm{MAPbI}_{3}$ states, an ideal situation for hole extraction. In contrast, negative values lead to an unfavourable interface for solar cell applications. Our calculations imply the presence of $\mathrm{MAPbI}_{3}$ states above those of $\mathrm{NiO}$ (Figure 1c) pointing to a valence band energy level alignment that is detrimental for hole collection. This is confirmed by a layer resolved projected density of states (PDOS, Figure 3a). Indeed, from the PDOS, one can extract the position of the VBM of the layers emulating the bulk of both materials $\left({ }^{\mathrm{Ni}} \mathrm{L}_{3}\right.$ for $\mathrm{NiO}$ and ${ }^{\mathrm{Pb}} \mathrm{L}_{4}$ for $\mathrm{MAPbI}_{3}$, Figure 1a). In the case of the $\mathrm{MAPbI}_{3} / \mathrm{NiO}$ interface, from the PDOS we obtain a VBO of about $-0.41 \mathrm{eV}$ between $\mathrm{MAPbI}_{3}$ and $\mathrm{NiO}$ (Figure 3). We obtain a similar VBO $(-0.36 \mathrm{eV})$ using the Hartree potential alignment ${ }^{46}$ (Text S4 and Figure S5, SI) showing that both the PDOS and the Hartree potential alignment methods can be used to compute band offsets. ${ }^{45,47}$ Surprisingly, the sign of our calculated VBO value seemingly contradicts the experimental findings, which point to an almost ideal VBO (ranging from +0.0 to $+0.4 \mathrm{eV}$ ) between $\mathrm{MAPbI}_{3}$ and $\mathrm{NiO}$ making the latter a suitable material for hole extraction and transport. ${ }^{14,18,48,49}$ 


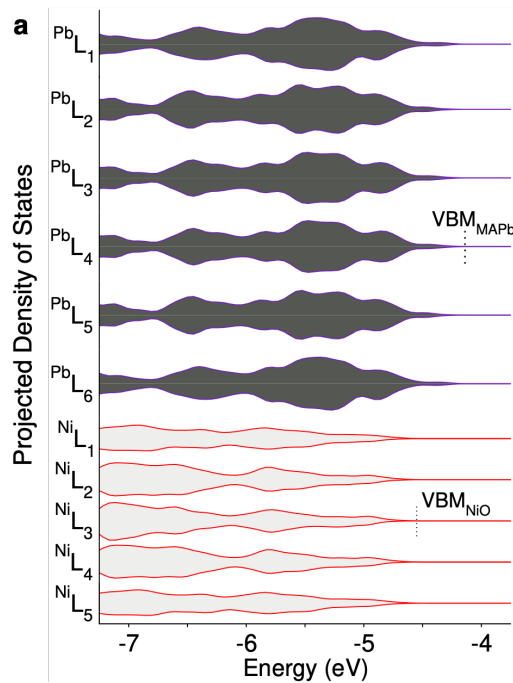

b Valence band offset

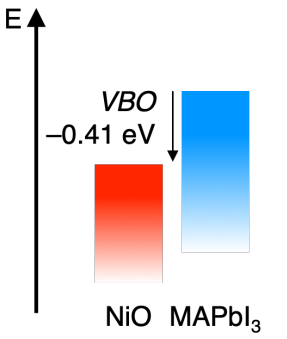

Figure 3: Valence band alignment in the $\mathrm{NiO} / \mathrm{MAPbI}_{3}$ pristine interface. a Layer-by-layer projected density of states (PDOS) of the relaxed interface model affording valence band alignment from the bulk-like parts of $\mathrm{MAPbI}_{3}$ and $\mathrm{NiO}$. $\mathbf{b}$ The valence band offset (VBO) determined from the PDOS. The computed VBO is not favourable for hole extraction.

Since this finding contrasts to experimental results, we checked that it was neither an artifact of the choice of the starting orthorhombic structure nor the considered number of layers in $\mathrm{MAPbI}_{3}$ or our computational approach. Firstly, we obtained similar results with tetragonal $\mathrm{MAPbI}_{3}$ as the starting structure of the interface (Text S5 and Figure S6, SI) and by increasing the number of layers in both $\mathrm{MAPbI}_{3}$ and $\mathrm{NiO}$ (Text $\mathrm{S} 6$ and Figure S7, $\mathrm{SI})$. Then, we recalculated the electronic structure using the GGA $+U$ formalism in planewave basis sets instead of localized basis sets (Text S7, SI). These new simulations lead to a negative $\mathrm{VBO}$ of $-0.53 \mathrm{eV}$, confirming the counterintuitive result. Hybrid functional calculations (HSE03 ${ }^{50}$ and HSE06 ${ }^{51}$ ) also confirm the trend $(\mathrm{VBO} \approx-0.70 \mathrm{eV})$. Thus, the sign of VBO is not an artifact of the Hubbard correction approach (Text S8, SI).

While the DFT approach is not responsible for the sign of the VBO, it might be influenced by the structural choices made in the construction of $\mathrm{NiO} / \mathrm{MAPbI}_{3}$ interface. Indeed, despite that STM experiments point to a MAI-termination in $\mathrm{MAPbI}_{3},{ }^{36} \mathrm{PbI}$ termination may not be completely excluded as it is reported to be slightly more stable by other first-principles simulations. ${ }^{52,53}$ To assess the possible effect of this termination on the electronic structure of 
the $\mathrm{NiO} / \mathrm{MAPbI}_{3}$ interface model, we considered the same interface but with $\mathrm{PbI}$ termination (Text S9 and Figure S11, SI). We find that the most stable configuration presents a VBO shift of $-0.15 \mathrm{eV}$ with $\mathrm{MAPbI}_{3}$ states above those of $\mathrm{NiO}$ indicative of an unfavorable alignment for hole collection. We note that in real thin films, several interface configurations may co-exist depending on the deposition technique and conditions. This could eventually lead to the formation of some metastable domains in the device, thus affecting the interfacial energetics (Figure S12, SI). However, we hypothesize that the thermodynamics of the system should make the favorable energy configurations to dominate.

Finally, considering the effect of substituting the cation in the perovskite layer, we also inspected the energy level alignment of $\mathrm{FAPbI}_{3} / \mathrm{NiO}$ interface (Text $\mathrm{S} 10, \mathrm{SI}$ ) with FA referring to Formamidinium $\left(\mathrm{FA}=\mathrm{CH}\left(\mathrm{NH}_{2}\right)_{2}\right)$. Here, we used the (001) surface of cubic $\mathrm{FAPbI}_{3}$ with FAI termination and lattice-matched it to $\mathrm{NiO}$, the latter being the substrate. We calculated a $\mathrm{VBO}$ of $-0.15 \mathrm{eV}$ with $\mathrm{FAPbI}_{3}$ states above those of $\mathrm{NiO}$ (Figure S13, SI). As in $\mathrm{MAPbI}_{3}$, this VBO is not optimal for hole collection from $\mathrm{FAPbI}_{3}$ to $\mathrm{NiO}$. However, the absolute value of this shift is lower as compared to $-0.41 \mathrm{eV}$ obtained with $\mathrm{MAPbI}_{3}$. Detailed information on this interface, the related discussion on the lower VBO with $\mathrm{FAPbI}_{3}$ and possible effects of mixed cation HOP on the properties of this interface can be found in Text S10 (SI). In the end, all these results confirm a valence band energy level alignment that is not in favour of efficient hole collection when considering defect-free interfaces of pristine materials.

Hence, a legitimate question about the possible origin of this apparent contradiction arises. Could it be related to the assumption of the defect-free $\mathrm{NiO}$ and $\mathrm{MAPbI}_{3}$ lattices in our model? What is the role of doping in the experimentally reported VBO? In the following, we aim to address these questions.

Role of vacancies Despite hybrid perovskites being referred to as "defect-tolerant", ${ }^{54-56}$ various defects are abundant in these materials and their role on the interfacial properties of 

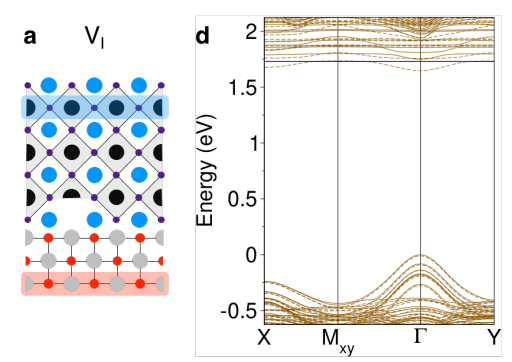

g Valence band offset
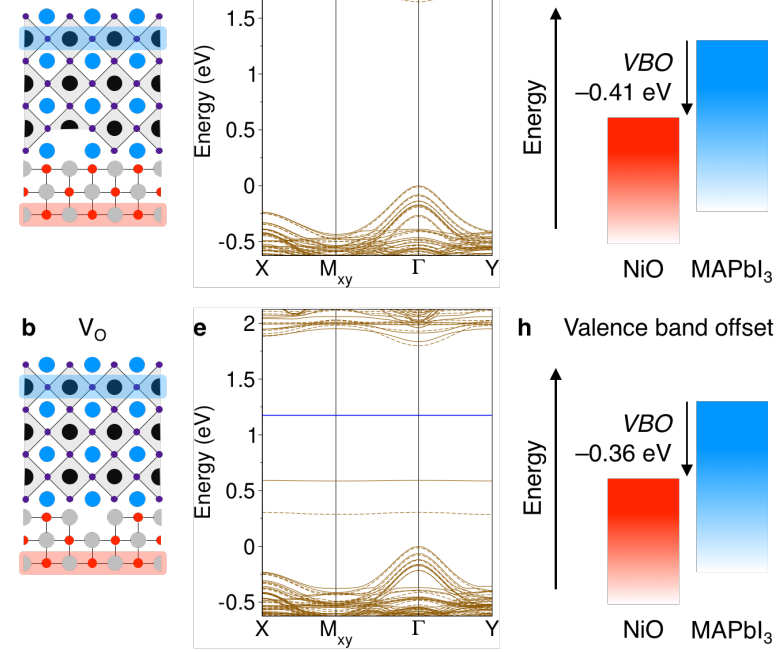

h Valence band offset
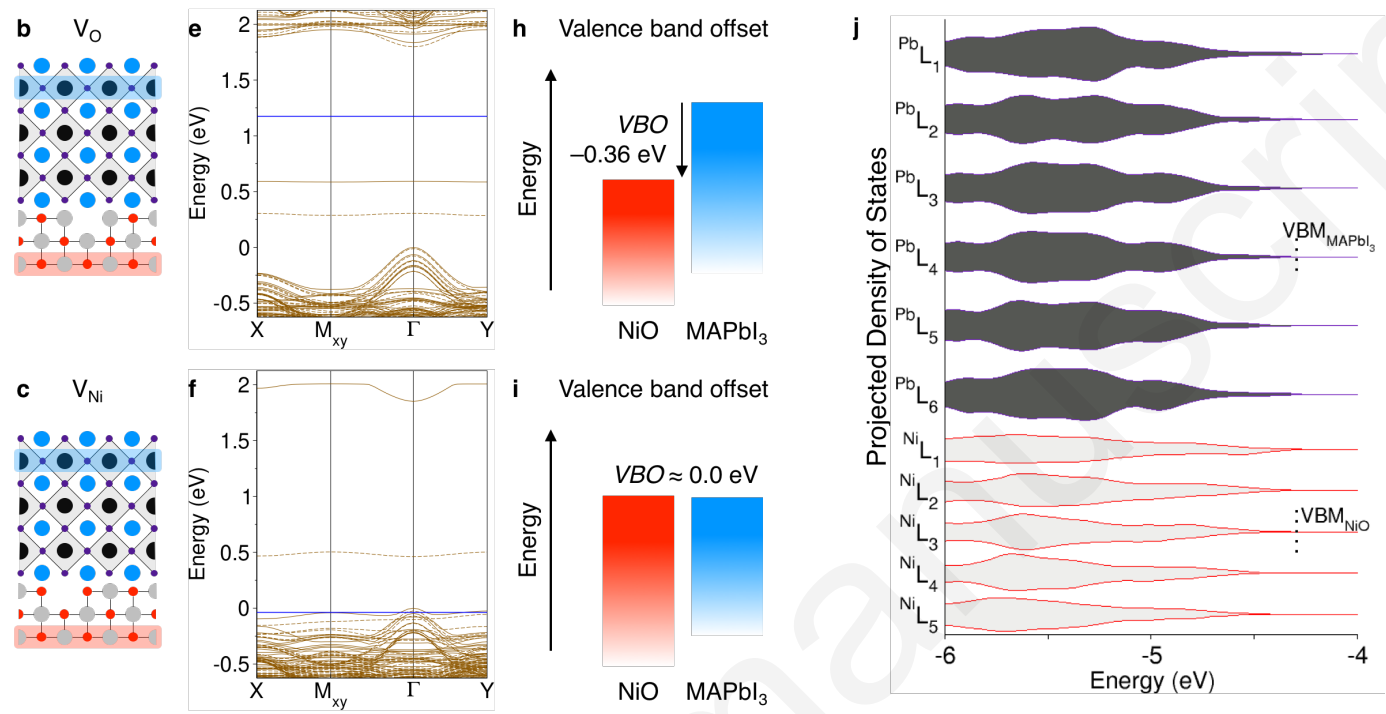

Figure 4: Role of vacancies in the $\mathrm{NiO} / \mathrm{MAPbI}_{3}$ interface. $\mathbf{a}, \mathbf{b}$, $\mathbf{c}$ Iodine $\left(\mathrm{V}_{\mathrm{I}}\right)$, oxygen $\left(\mathrm{V}_{\mathrm{O}}\right)$ and nickel $\left(\mathrm{V}_{\mathrm{Ni}}\right)$ vacancy models, respectively. $\mathbf{d}, \mathbf{e}, \mathbf{f}$ Corresponding calculated band structures. The valence band maximum at $\Gamma$ point is taken as the energy reference, a blue line marks the Fermi level. Plain and dashed lines correspond to majority and minority spins, respectively. $\mathbf{g}, \mathbf{h}, \mathbf{i}$ Corresponding computed valence band offsets. $\mathbf{j}$ Layer by layer projected density of states (PDOS) of the interface model with $\mathrm{V}_{\mathrm{Ni}}$ resulting in the $\mathrm{VBO}$ shown in i. Nickel vacancies produce a valence band alignment favourable to hole extraction.

$\mathrm{MAPbI}_{3}$ remains elusive. While it is of great fundamental and technological interest, there is no theoretical study of their effects on the electronic structure of $\mathrm{NiO} / \mathrm{MAPbI}_{3}$ interface, which we address in this section. To introduce defects and later dopants into the interface model, we enlarge the defect-free cell in order to reduce the effects of spurious interactions, originating from the periodic boundary conditions, on the computed properties (see Text S11, SI). We consider the impact of different defects that could potentially influence the valence band alignment: iodine vacancies $\left(\mathrm{V}_{\mathrm{I}}\right.$, Figure $\left.4 \mathrm{a}\right)$, oxygen vacancies $\left(\mathrm{V}_{\mathrm{O}}\right.$, Figure $\left.4 \mathrm{~b}\right)$ and nickel vacancies $\left(\mathrm{V}_{\mathrm{Ni}}\right.$, Figure $\left.4 \mathrm{c}\right)$. Furthermore, $\mathrm{MA}$ vacancies $\left(\mathrm{V}_{\mathrm{MA}}\right), \mathrm{Pb}$ vacancies $\left(\mathrm{V}_{\mathrm{Pb}}\right)$ and Schottky-type defect MAI vacancies ( $\mathrm{V}_{\mathrm{MAI}}$ ) are presented in the SI (Text S11, SI). 
The band structure of $\mathrm{V}_{\mathrm{I}}$ (Figure 4d) shows a shallow defect level at the conduction band, in agreement with previous results, ${ }^{57}$ while having negligible effect on the valence band. In the case of $\mathrm{V}_{\mathrm{O}}$, localized mid-gap trap states are formed. The analysis of the wavefunctions shows that these states arise from $\mathrm{Ni}$ atoms in direct contact with the vacant oxygen site. Such mid-gap states are a source of non-radiative recombinations detrimental to photovoltaic performances. However, similar to $\mathrm{V}_{\mathrm{I}}$, the presence of $\mathrm{V}_{\mathrm{O}}$ brings no change to the shape of the valence bands. Both $\mathrm{V}_{\mathrm{I}}$ and $\mathrm{V}_{\mathrm{O}}$ barely affect the $\mathrm{VBO}$ of the interface model as compared to the defect-free cell and we obtain a negative value of $c a .-0.40 \mathrm{eV}$ for the two systems (Figure 4g,h).

Similarly, we calculate a VBO of $c a$. $-0.31 \mathrm{eV}$ for $\mathrm{V}_{\mathrm{MAI}}$ with the valence band barely affected (Figure $\mathrm{S} 15, \mathrm{SI}) . \mathrm{V}_{\mathrm{MA}}$ and $\mathrm{V}_{\mathrm{Pb}}$ at the interface region $\left({ }^{\mathrm{Pb}} \mathrm{L}_{6}\right.$ ) present a $\mathrm{VBO}$ of $-0.05 \mathrm{eV}$ and $-0.10 \mathrm{eV}$, respectively and the Fermi level is shifted to the valence bands forming shallow acceptor states in agreement with previous reports (Figures S16-S19, SI). ${ }^{58,59}$ In the bulk-like region of $\mathrm{MAPbI}_{3}\left({ }^{\mathrm{Pb}} \mathrm{L}_{4}\right), \mathrm{VBO}$ of $\mathrm{V}_{\mathrm{MA}}$ and $\mathrm{V}_{\mathrm{Pb}}$ become $c a$. $-0.46 \mathrm{eV}$ and ca. $-0.41 \mathrm{eV}$, respectively (Figures S17 and S19, SI). Hence with $\mathrm{MAPbI}_{3}$ related defects along with $\mathrm{V}_{\mathrm{O}}$, VBO remain essentially unfavorable for hole collection across $\mathrm{NiO} / \mathrm{MAPbI}_{3}$ interface.

On the other hand, the presence of a $\mathrm{V}_{\mathrm{Ni}}$ site at both the interface and bulk-like layers $\left({ }^{\mathrm{Ni}} \mathrm{L}_{1}\right.$ and ${ }^{\mathrm{Ni}} \mathrm{L}_{3}$ ), is accompanied by the emergence of less dispersive bands (Figure $4 \mathrm{f}$ and Figure $\mathrm{S} 20, \mathrm{SI})$ that are localized on $\mathrm{O}$ atoms (2p states) around $\mathrm{V}_{\mathrm{Ni}}$ and the next neighbor $\mathrm{Ni}$ atoms (3d states). Noteworthy, it results in a large positive energy shift of the valence bands related to $\mathrm{NiO}$, which becomes almost equals to the $\mathrm{VBM}$ of the $\mathrm{MAPbI}_{3}$ (Figure $4 \mathrm{f}$ and Figures S20-S22, SI). As a consequence, the VBO becomes almost vanishing (Figure 4i,j) and the material system becomes p-type (Fermi energy crosses the valence states). The latter result is in agreement with the valence band alignments given in the literature between $\mathrm{MAPbI}_{3}$ and $\mathrm{NiO} .{ }^{14,49} \mathrm{It}$ is known that $\mathrm{V}_{\mathrm{Ni}}$ makes $\mathrm{NiO}$ become a $p$-type semi-conductor, ${ }^{60,61}$ but its effect on the interfacial properties of $\mathrm{MAPbI}_{3}$ has not been assessed previously. This 
Experimental confirmation The reported experimental energy level alignments between $\mathrm{NiO}$ and $\mathrm{MAPbI}_{3}$ generally, if not all, rely on the difference between the work functions $\left(\mathrm{W}_{F}\right)$ of $\mathrm{NiO}$ and the absolute valence band energy (AVBE) of $\mathrm{MAPbI}_{3}$. While the AVBE of $c a$. $-5.4 \mathrm{eV}$ for $\mathrm{MAPbI}_{3}$ seems more consensual in the realm of reported data, ${ }^{63}$ the measured $\mathrm{W}_{F}$ for $\mathrm{NiO}$ largely depends on the processing conditions and $\mathrm{NiO}$ surface exposure. ${ }^{64}$ Reported $\mathrm{W}_{F}$ values fall between $-5.0 \mathrm{eV}$ to $-5.6 \mathrm{eV},{ }^{5,7,64-66}$ reaching as high as $-6.2 \mathrm{eV}$ to $-6.7 \mathrm{eV}$ in pure $\mathrm{NiO}$ film deposited in-situ. ${ }^{64}$ For a purely processed $\mathrm{NiO}$ film, its $\mathrm{W}_{F}$ can be assumed to be close in value to its AVBE as its purity makes it stoichiometric with no defect. Indeed, the measured UPS absolute valence band energy of $-6.5 \mathrm{eV}^{67,68}$ for $\mathrm{NiO}$ agrees well with the $\mathrm{W}_{F}$ of purely deposited $\mathrm{NiO}$. Hence, using an AVBE of $-6.2 \mathrm{eV}$ to $-6.5 \mathrm{eV}$ for $\mathrm{NiO}$ to align it with $\mathrm{MAPbI}_{3}$, the resulting $\mathrm{VBO}$ gives $-0.8 \mathrm{eV}$ to $-1.1 \mathrm{eV}$, which is in a good agreement with our prediction for the defect-free $\mathrm{NiO} / \mathrm{MAPbI}_{3}$ interface model (Figure $3 \mathrm{~b}$ ). Moreover, considering $\mathrm{W}_{F}$ of $\mathrm{NiO}$ in air-exposed conditions $(c a .-5.4 \mathrm{eV}$ ), with potential defects like Ni vacancies, ${ }^{62}$ we recover the literature reported near-ideal valence band alignment between $\mathrm{NiO}$ and $\mathrm{MAPbI}_{3}$. This is in a good agreement with our predictions on the role of $\mathrm{Ni}$ vacancies in turning $\mathrm{NiO}$ into a $p$-type material in the interface model. Therefore, we believe that the experimentally reported deposited $\mathrm{NiO}$ films via sputtering/spin-coating during HOP fabrication present dominant Ni vacancies, ${ }^{62}$ which appear to help the performances of nickel-based perovskite solar cells. However, the appearance of the less-dispersive mid-gap defect-like states due to the presence of $\mathrm{V}_{\mathrm{Ni}}$ at the interface can alleviate the efficiency of hole carrier collection at the $\mathrm{NiO} / \mathrm{MAPbI}_{3}$ interface. Since all the investigated defects, except $\mathrm{V}_{\mathrm{Ni}}$, lead to a negative $\mathrm{VBO}$, we postulate that increasing their concentration 
would only worsen the properties of this interface. Therefore, it is desirable to limit and/or reduce their concentration inside the film while, at the same time, preserving an ideal energy alignment. A practical strategy to achieve that goal is by doping, which is discussed in the next section.

Role of doping Similar to other semiconductors, doping strategies have been used to finetune the properties of $\mathrm{NiO}$, notably using Li for improved $p$-conductivity ${ }^{69,70}$ and solar efficiencies in perovskites. ${ }^{18,71}$ To bring more fundamental understanding to this improvement, we study the effect of $\mathrm{Li}$ doping of $\mathrm{NiO}$ by considering the substitution in our simulation cell of $2 \mathrm{Ni}$ atoms, chosen as far from each other as possible, by $2 \mathrm{Li}$ atoms (Figure 5a). The doping is done at the interface region of $\mathrm{NiO}\left({ }^{\mathrm{Ni}} \mathrm{L}_{1}\right.$, Figure $\left.5 \mathrm{~b}\right)$ in direct contact with $\mathrm{MAPbI}_{3}$ and in the bulk-like region of $\mathrm{NiO}\left({ }^{\mathrm{Ni}} \mathrm{L}_{3}\right.$, Figure 1c). Li doping at the interface-like region is $1.5 \mathrm{eV}(0.75 \mathrm{eV}$ per $\mathrm{Li}$ atom $)$ more favorable than in the bulk-like region. Hence, our results indicate that the doped $\mathrm{Li}$ atoms would probably be distributed in $\mathrm{NiO}$ at the interface region being in contact with $\mathrm{MAPbI}_{3}$. Nevertheless, the other configurations cannot be completely excluded given the relatively low energy difference for the system size modeled.

The band structures of ' $\mathrm{Li}$ at the interface' (Figure 5d) and 'Li in the bulk' (Figure 5e) region show that Li doping facilitates a $p$-type conduction, while no localized band is created in the gap. The reorganization of the valence bands under the influence of Li, as shown by the PDOS (Figures S23 and S24, SI), leads to an improved VBO value that becomes slightly positive $(+0.00 \mathrm{eV}$ for $\mathrm{Li}$ at the 'interface' region and $+0.05 \mathrm{eV}$ at the 'bulk' one, Figure $5 \mathrm{f}, \mathrm{g})$. Therefore, Li doping recovers an ideal valence energy alignment obtained with $\mathrm{V}_{\mathrm{Ni}}$ while suppressing the presence of non-dispersive mid-gap states. Since dopants are not always evenly distributed in the materials, we also consider a model in which the 2 substituted $\mathrm{Ni}$ atoms are only separated by one O atom (Figures S23 and S24, SI). This configuration could be viewed as a well controlled $\mathrm{NiO}$ doped system with a high concentration of $\mathrm{Li}$ in a confined region of the material. Energetically, the original 'spread' model is $0.3 \mathrm{eV}(0.15 \mathrm{eV}$ per $\mathrm{Li}$ 


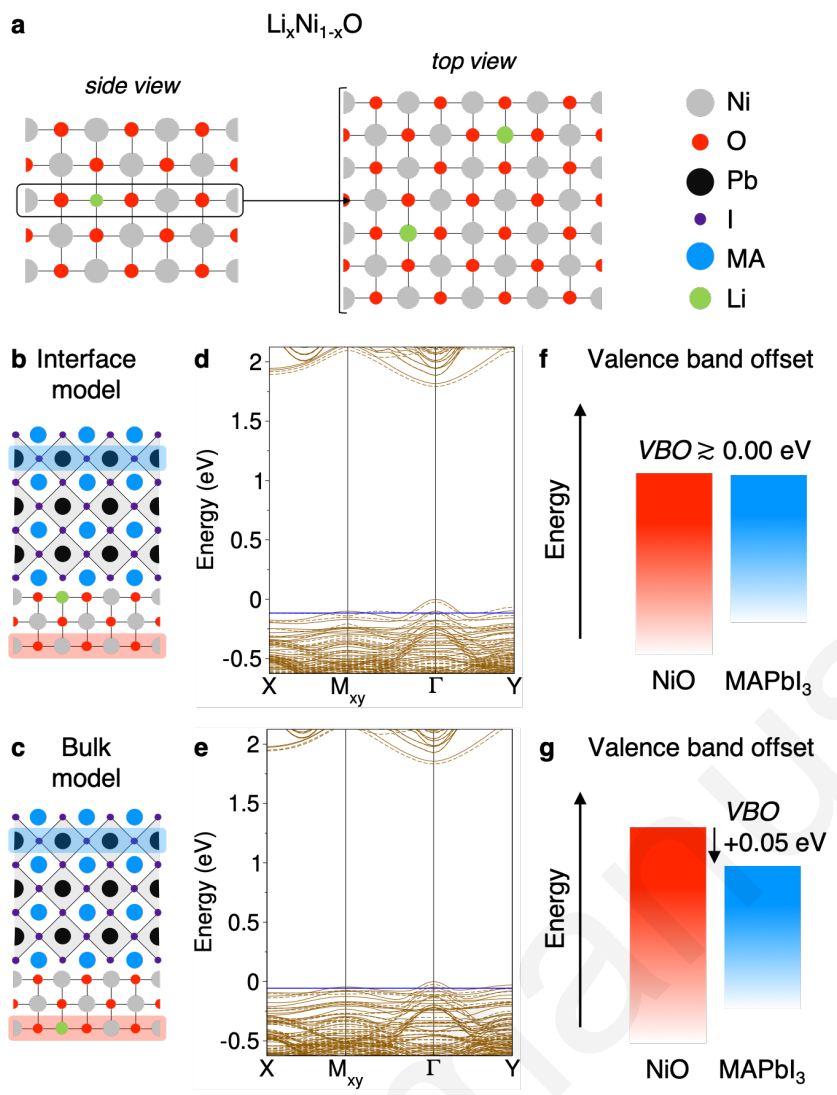

Figure 5: Role of $\mathrm{Li}$ doping in the $\mathrm{NiO} / \mathrm{MAPbI}_{3}$ interface. a $\mathrm{Li}_{x} \mathrm{Ni}_{1-x} \mathrm{O}$ models with dopants spread across a NiO layer. b 'Interface' model where the doped layer is at the interface. c 'Bulk' model where the dopants are placed in the bulk-like layer. Calculated band structures of the $\mathbf{d}$ 'interface' and e 'bulk' models. The valence band maximum at the $\Gamma$ point is taken as the energy reference, a blue line marks the Fermi level. Plain and dashed lines correspond to majority and minority spins, respectively. f, $\mathbf{g}$ Corresponding computed valence band offsets. Both situations lead to a favorable hole extraction.

atom) more favorable than the clustered one at both the interface-like and bulk-like regions. We notice that VBO shift increases when Li atoms are clustered in a confined space resulting in $+0.31 \mathrm{eV}$ and $+0.26 \mathrm{eV}$ values at the interface-like and bulk-like regions, respectively. We also examined the effect of increased dopant concentration by substituting $4 \mathrm{Ni}$ atoms by $\mathrm{Li}$ atoms, bringing the concentration to 5\%. Again, we examined configurations where Li atoms are 'clustered' in one layer and spread across two NiO layers (Figure S25, SI). Here, VBO varies from $+0.21 \mathrm{eV}$ when $\mathrm{Li}$ atoms are"clustered" to $+0.15 \mathrm{eV}$ when they are 'spread' (Figure S25, SI). This suggests that $5 \% \mathrm{Li}$ content in $\mathrm{NiO}$ should improve the conversion efficiency of the HOP device as a result of increased open circuit voltage. 
The calculated improved energy level alignment of $\mathrm{NiO}$ doped $\mathrm{Li}$ with $\mathrm{MAPbI}_{3}$ is in agreement with the enhanced efficiency of $\mathrm{LiNiO}_{\mathrm{x}} / \mathrm{MAPbI}_{3}$ devices as compared to undoped NiO. ${ }^{18,71}$ The PDOS of the different systems with Li doping shows that the introduction of $\mathrm{Li}$ pushes $\mathrm{NiO}$ states to the same energy level or above those of $\mathrm{MAPbI}_{3}$ while reinforcing their hybridization at the valence band maximum (Figures S26, SI). At the same time, Li states remain about $0.5 \mathrm{eV}$ below (Figure S26, SI). The net effect results in a doped $\mathrm{NiO}$ being a better hole transport layer.

Interestingly, we obtained an increased $\mathrm{VBO}(\geq 0.00 \mathrm{eV})$ of $\mathrm{NiO} / \mathrm{MAPbI}_{3}$ heterostructure when $\mathrm{NiO}$ is doped with $\mathrm{Rb}$ and Cs atoms (Figures S27 and S28, SI). As with Li, their insertion into $\mathrm{NiO}$ enhances the hybridization between $\mathrm{MAPbI}_{3}$ and the HTL. With Rb doping, we calculated a VBO of $+0.46 \mathrm{eV}$ (Figure S27, SI). For Cs, we calculated a VBO of $+0.41 \mathrm{eV}$ (Figure S28, SI). The large atomic size of Rb and Cs induces more structural relaxation at the interface causing an increase of VBO. Given that Cs and Rb are already adopted as cations in mixed-cation $3 \mathrm{D}$ HOP,${ }^{16,33}$ they may be considered as alternative dopants of $\mathrm{NiO}$ in the search of optimizing the efficiency of $\mathrm{MAPbI}_{3}$ solar devices.

\section{Conclusions}

In summary, we demonstrate the role of native defects and doping on the energetics and charge carrier extraction for devices based on $\mathrm{NiO}$ interfaces. Our computed valence band offsets are consistent with experimental UPS absolute valence band energies of NiO. Moreover, our analysis clarifies the experimentally reported ideal valence band alignment between $\mathrm{MAPbI}_{3}$ and $\mathrm{NiO}$, which is related to the intrinsic Ni vacancies of the hole transporting material. The appearance of these dominant Ni-vacancies is commensurate with experimental report on standard $\mathrm{NiO}$ processing conditions. ${ }^{62}$ Furthermore, we highlight the effect of Li doping in $\mathrm{NiO}$ to reach improved energy alignment of the perovskite-NiO interface fostering enhanced hole extraction. Hence, we propose alternatives to $\mathrm{Li}$ doping of $\mathrm{NiO}$ such as $\mathrm{Cs}$ or $\mathrm{Rb}$ to further fine-tune the $\mathrm{NiO}$ interfacial energetics. Additionally, we highlight 
the importance of the dielectric mismatch. For instance, the dielectric properties related to the high-frequency component of the interface indicate a low dielectric mismatch between $\mathrm{MAPbI}_{3}$ and $\mathrm{NiO}$, which benefits hole collection. The combined results bring together a holistic picture on the interfacial properties of $\mathrm{NiO}$. Our findings open avenues for the optimization of thin-film devices based on $\mathrm{NiO}$ contacts, and help the reader to adopt relevant theoretical strategies to model the properties of heterostructures.

\section{Supporting Information Available}

Computational details; Electronic structure of NiO; Detailed description of the construction of the interface models; Energy level alignment using Hartree potential profiles; Additional plane-wave calculations on valence band energy level alignments; Effects of $\mathrm{MAPbI}_{3} \mathrm{surface}$ termination and substituting MA cation on the valence band offset of $\mathrm{MAPbI}_{3} / \mathrm{NiO}$; Projected density of states for systems with vacancies or dopants; Band structures for systems with dopants;

\section{Acknowledgement}

ISCR and FOTON have received funding from the European Union's Horizon 2020 program, through a FET Open research and innovation action under the grant agreement No 687008. The work at the Institut des Sciences Chimiques de Rennes was supported by Agence Nationale pour la Recherche (TRANSHYPERO project). J.E. acknowledges financial support from the Institute Universitaire de France. This work was performed, in part, at the Center for Integrated Nanotechnologies, a U.S. Department of Energy, Office of Science User Facility. S.T. acknowledges support of LANL LDRD program. This work was granted access to the HPC resources of [TGCC/CINES/IDRIS] under the allocations 2016-x2016097682/2017A0010907682/2018-A0010907682 made by GENCI. 


\section{References}

(1) Sherkar, T. S.; Koster, L. J. A. Dielectric Effects at Organic/Inorganic Interfaces in Nanostructured Devices. ACS Appl. Mater. Interfaces 2015, 7, 11881-11889.

(2) Schulz, P.; Cahen, D.; Kahn, A. Halide Perovskites: Is It All about the Interfaces? Chem. Rev. 2019, 119, 3349-3417.

(3) Chueh, C.-C.; Li, C.-Z.; Jen, A. K.-Y. Recent Progress and Perspective in Solution-Processed Interfacial Materials for Efficient and Stable Polymer and Organometal Perovskite Solar Cells. Energy Environ. Sci. 2015, 8, 1160-1189.

(4) Yip, H.-L.; Jen, A. K.-Y. Recent Advances in Solution-Processed Interfacial Materials For Efficient And Stable Polymer Solar Cells. Energy Environ. Sci. 2012, 5, 5994-6011.

(5) Steirer, K. X.; Chesin, J. P.; Widjonarko, N. E.; Berry, J. J.; Miedaner, A.; Ginley, D. S.; Olson, D. C. Solution Deposited NiO Thin-Films As Hole Transport Layers in Organic Photovoltaics. Org. Elec. 2010, 11, 1414-1418.

(6) He, J.; Lindström, H.; Hagfeldt, A.; Lindquist, S.-E. Dye-Sensitized Nanostructured Tandem Cell-First Demonstrated Cell With a Dye-Sensitized Photocathode. Sol. Energy Mater Sol. Cells 2000, 62, 265-273.

(7) Irwin, M. D.; Buchholz, D. B.; Hains, A. W.; Chang, R. P. H.; Marks, T. J. p-Type Semiconducting Nickel Oxide As An Efficiency-Enhancing Anode Interfacial Layer In Polymer Bulk-Heterojunction Solar Cells. Proc. Natl. Acad. Sci. U.S.A. 2008, 105, 2783-2787.

(8) Sato, H.; Minami, T.; Takata, S.; Yamada, T. Transparent Conducting p-Type NiO Thin Films Prepared By Magnetron Sputtering. Thin Solid Films 1993, 236, 27-31.

(9) Kato, H.; Asakura, K.; Kudo, A. Highly Efficient Water Splitting into $\mathrm{H}_{2}$ and $\mathrm{O}_{2}$ over Lanthanum-Doped $\mathrm{NaTaO}_{3}$ Photocatalysts with High Crystallinity and Surface Nanostructure. J. Am. Chem. Soc. 2003, 125, 3082-3089.

(10) Varghese, B.; Reddy, M. V.; Yanwu, Z.; Lit, C. S.; Hoong, T. C.; Subba Rao, G. V.; Chowdari, B. V. R.; Wee, A. T. S.; Lim, C. T.; Sow, C.-H. Fabrication of NiO Nanowall Electrodes for High Performance Lithium Ion Battery. Chem. Mater. 2008, 20, 3360-3367.

(11) https://www.nrel.gov/pv/cell-efficiency.html, Accessed: 15-10-2019.

(12) You, J.; Meng, L.; Song, T.-B.; Guo, T.-F.; Yang, Y. M.; Chang, W.-H.; Hong, Z.; Chen, H.; Zhou, H.; Chen, Q.; Liu, Y.; De Marco, N.; Yang, Y. Improved Air Stability of Perovskite Solar Cells Via Solution-Processed Metal Oxide Transport Layers. Nature Nanotech. 2016, $11,75-81$.

(13) Chen, W.; Wu, Y.; Yue, Y.; Liu, J.; Zhang, W.; Yang, X.; Chen, H.; Bi, E.; Ashraful, I.; Grätzel, M.; Han, L. Efficient and Stable Large-Area Perovskite Solar Cells with Inorganic Charge Extraction Layers. Science 2015, 350, 944-948.

(14) Seo, S.; Park, I. J.; Kim, M.; Lee, S.; Bae, C.; Jung, H. S.; Park, N.-G.; Kim, J. Y.; Shin, H. An Ultra-Thin, Un-Doped NiO Hole Transporting Layer of Highly Efficient (16.4\%) OrganicInorganic Hybrid Perovskite Solar Cells. Nanoscale 2016, 8, 11403-11412. 
(15) Bush, K. A. et al. 23.6\%-Efficient Monolithic Perovskite/Silicon Tandem Solar Cells with Improved Stability. Nature Energy 2017, 2, 17009.

(16) Tsai, H.; Asadpour, R.; Blancon, J.-C.; Stoumpos, C. C.; Durand, O.; Strzalka, J. W.; Chen, B.; Verduzco, R.; Ajayan, P. M.; Tretiak, S.; Even, J.; Alam, M. A.; Kanatzidis, M. G.; Nie, W.; Mohite, A. D. Light-Induced Lattice Expansion leads to High-Efficiency Perovskite Solar Cells. Science 2018, 360, 67-70.

(17) Chen, W.; Wu, Y.; Liu, J.; Qin, C.; Yang, X.; Islam, A.; Cheng, Y.-B.; Han, L. Hybrid Interfacial Layer Leads To Solid Performance Improvement of Inverted Perovskite Solar Cells. Energy Environ. Sci. 2015, 8, 629-640.

(18) Nie, W.; Tsai, H.; Blancon, J.-C.; Liu, F.; Stoumpos, C. C.; Traore, B.; Kepenekian, M.; Durand, O.; Katan, C.; Tretiak, S.; Crochet, J.; Ajayan, P. M.; Kanatzidis, M.; Even, J.; Mohite, A. D. Critical Role of Interface and Crystallinity on the Performance and Photostability of Perovskite Solar Cell on Nickel Oxide. Adv. Mater. 2018, 30, 1703879.

(19) Mosconi, E.; Ronca, E.; De Angelis, F. First-Principles Investigation of the $\mathrm{TiO}_{2} /$ Organohalide Perovskites Interface: The Role of Interfacial Chlorine. J. Phys. Chem. Lett. 2014, 5, 26192625.

(20) Li, L.; Mi, J.; Yong, Y.; Mao, B.; Shi, W. First-Principles Study On the Lattice Plane And Termination Dependence of the Electronic Properties of the $\mathrm{NiO} / \mathrm{CH}_{3} \mathrm{NH}_{3} \mathrm{PbI}_{3}$ Interfaces. $J$. Mater. Chem. C 2018, 6, 8226-8233.

(21) Du Plessis, P. de V.; van Tonder, S. J.; Alberts, L. Elastic Constants of a NiO Single Crystal: I. J. Phys. C: Solid State Phys. 1971, 4, 1983-1987.

(22) Li, J.-L.; Rignanese, G.-M.; Louie, S. G. Quasiparticle Energy Bands of NiO in the $G W$ Approximation. Phys. Rev. B 2005, 71, 193102.

(23) Jiang, H.; Gomez-Abal, R. I.; Rinke, P.; Scheffler, M. First-Principles Modeling of Localized $d$ States with the GW/LDA+U Approach. Phys. Rev. B 2010, 82, 045108.

(24) Panda, S. K. et al. High Photon Energy Spectroscopy of NiO: Experiment and Theory. Phys. Rev. B 2016, 93, 235138.

(25) Rohrbach, A.; Hafner, J.; Kresse, G. Molecular Adsorption on the Surface of Strongly Correlated Transition-Metal Oxides: A Case Study For CO/NiO(100). Phys. Rev. B 2004, 69, 075413 .

(26) Anisimov, V. I.; Aryasetiawan, F.; Lichtenstein, A. I. First-Principles Calculations of the Electronic Structure And Spectra of Strongly Correlated Systems: the LDA+U Method. J. Phys.: Condens. Matter 1997, 9, 767-808.

(27) Dudarev, S. L.; Botton, G. A.; Savrasov, S. Y.; Humphreys, C. J.; Sutton, A. P. ElectronEnergy-Loss Spectra And the Structural Stability of Nickel Oxide: An LSDA+U Study. Phys. Rev. B 1998, 57, 1505-1509.

(28) Soler, M.; Artacho, E.; Gale, J. D.; Garc, A.; Junquera, J.; Ordejon, P.; Daniel, S. The SIESTA Method for Ab Initio Order-N Materials. J. Phys.: Condens. Matter 2002, 14, 2745-2779. 
(29) Artacho, E.; Anglada, E.; Diéguez, O.; Gale, J. D.; García, A.; Junquera, J.; Martin, R. M.; Ordejón, P.; Pruneda, J. M.; Sánchez-Portal, D.; Soler, J. M. The SIESTA Method; Developments and Applicability. J. Phys.: Condens. Matter 2008, 20, 064208.

(30) Cococcioni, M.; de Gironcoli, S. Linear Response Approach to the Calculation of the Effective Interaction Parameters in the LDA + U Method. Phys. Rev. B 2005, 71, 035105.

(31) Ködderitzsch, D.; Hergert, W.; Temmerman, W. M.; Szotek, Z.; Ernst, A.; Winter, H. Exchange Interactions in $\mathrm{NiO}$ and at the $\mathrm{NiO}(100)$ Surface. Phys. Rev. B 2002, 66, 064434.

(32) Welton-Cook, M. R.; Prutton, M. LEED Calculations for the NiO (100) Surface: Extension to Lower Energies. J. Phys. C: Solid State Phys. 1980, 13, 3993-3400.

(33) Saliba, M.; Matsui, T.; Domanski, K.; Seo, J.-Y.; Ummadisingu, A.; Zakeeruddin, S. M.; Correa-Baena, J.-P.; Tress, W. R.; Abate, A.; Hagfeldt, A.; Grätzel, M. Incorporation of Rubidium Cations Into Perovskite Solar Cells Improves Photovoltaic Performance. Science 2016, 354, 206-209.

(34) Yang, W. S.; Park, B.-W.; Jung, E. H.; Jeon, N. J.; Kim, Y. C.; Lee, D. U.; Shin, S. S.; Seo, J.; Kim, E. K.; Noh, J. H.; Seok, S. I. Iodide Management in Formamidinium-Lead-Halide-Based Perovskite Layers for Efficient Solar Cells. Science 2017, 356, 1376-1379.

(35) Even, J.; Carignano, M.; Katan, C. Molecular Disorder and Translation/Rotation Coupling in the Plastic Crystal Phase of Hybrid Perovskites. Nanoscale 2016, 8, 6222-6236.

(36) She, L.; Liu, M.; Zhong, D. Atomic Structures of $\mathrm{CH}_{3} \mathrm{NH}_{3} \mathrm{PbI}_{3}$ (001) Surfaces. ACS Nano 2016, 10, 1126-1131.

(37) Quarti, C.; Mosconi, E.; De Angelis, F. Interplay of Orientational Order and Electronic Structure in Methylammonium Lead Iodide: Implications for Solar Cell Operation. Chem. Mater. 2014, 26, 6557-6569.

(38) Wyckoff, R. W. G. Cadmium Chloride Structure, 2nd ed.; Interscience Publishers, New York, New York, 1963; Vol. 1; pp 239-444.

(39) Even, J.; Pedesseau, L.; Kepenekian, M. Electronic Surface States and Dielectric Self-Energy Profiles in Colloidal Nanoscale Platelets of CdSe. Phys. Chem. Chem. Phys. 2014, 16, 2518225190.

(40) Sapori, D.; Kepenekian, M.; Pedesseau, L.; Katan, C.; Even, J. Quantum Confinement and Dielectric Profiles of Colloidal Nanoplatelets of Halide Inorganic and Hybrid Organic-Inorganic Perovskites. Nanoscale 2016, 8, 6369-6378.

(41) Gielisse, P. J.; Plendl, J. N.; Mansur, L. C.; Marshall, R.; Mitra, S. S.; Mykolajewycz, R.; Smakula, A. Infrared Properties of $\mathrm{NiO}$ and $\mathrm{CoO}$ and Their Mixed Crystals. J. Appl. Phys. 1965, 36, 2446-2450.

(42) Hirasawa, M.; Ishihara, T.; Goto, T.; Uchida, K.; Miura, N. Magnetoabsorption of the Lowest Exciton in Perovskite-Type Compound $\left(\mathrm{CH}_{3} \mathrm{NH}_{3}\right) \mathrm{PbI}_{3}$. Physica B Condens. Matter. 1994, 201, 427-430. 
(47) Peressi, M.; Binggeli, N.; Baldereschi, A. Band Engineering at Interfaces: Theory And Numerical Experiments. J. Phys. D: Appl. Phys. 1998, 31, 1273-1299.

(48) Liu, T.; Chen, K.; Hu, Q.; Zhu, R.; Gong, Q. Inverted Perovskite Solar Cells: Progresses and Perspectives. Adv. Energy Mater. 2016, 6, 1600457.

(49) Niu, G.; Wang, S.; Li, J.; Li, W.; Wang, L. Oxygen Doping in Nickel Oxide For Highly Efficient Planar Perovskite Solar Cells. J. Mater. Chem. A 2018, 6, 4721-4728.

(50) Heyd, J.; Scuseria, G. E.; Ernzerhof, M. Hybrid Functionals Based on a Screened Coulomb Potential. J. Chem. Phys. 2003, 118, 8207-8215.

(51) Heyd, J.; Scuseria, G. E.; Ernzerhof, M. Erratum: "Hybrid Functionals Based on a Screened Coulomb Potential" [J. Chem. Phys. 118, 8207 (2003)]. J. Chem. Phys. 2006, 124, 219906.

(52) Haruyama, J.; Sodeyama, K.; Han, L.; Tateyama, Y. Termination Dependence of Tetragonal $\mathrm{CH}_{3} \mathrm{NH}_{3} \mathrm{PbI}_{3}$ Surfaces for Perovskite Solar Cells. J. Phys. Chem. Lett. 2014, 5, 2903-2909.

(53) Volonakis, G.; Giustino, F. Interfaces Between Graphene-Related Materials and $\mathrm{MAPbI}_{3}$ : Insights from First-Principles. Adv. Mater. Interfaces 2018, 5, 1800496.

(54) Steirer, K. X.; Schulz, P.; Teeter, G.; Stevanovic, V.; Yang, M.; Zhu, K.; Berry, J. J. Defect Tolerance in Methylammonium Lead Triiodide Perovskite. ACS Energy Lett. 2016, 1, 360-366.

(55) Meggiolaro, D.; De Angelis, F. First-Principles Modeling of Defects in Lead Halide Perovskites: Best Practices and Open Issues. ACS Energy Lett. 2018, 3, 2206-2222.

(56) Yavari, M. et al. How Far Does the Defect Tolerance of Lead-Halide Perovskites Range? The Example of Bi Impurities Introducing Efficient Recombination Centers. J. Mater. Chem. A 2019, 7, 23838-23853.

(57) Meggiolaro, D.; Motti, S. G.; Mosconi, E.; Barker, A. J.; Ball, J.; Andrea Riccardo Perini, C.; Deschler, F.; Petrozza, A.; De Angelis, F. Iodine Chemistry Determines the Defect Tolerance of Lead-Halide Perovskites. Energy Environ. Sci. 2018, 11, 702-713. 
(58) Kim, J.; Lee, S.-H.; Lee, J. H.; Hong, K.-H. The Role of Intrinsic Defects in Methylammonium Lead Iodide Perovskite. J. Phys. Chem. Lett. 2014, 5, 1312-1317.

(59) Kye, Y.-H.; Yu, C.-J.; Jong, U.-G.; Chen, Y.; Walsh, A. Critical Role of Water in Defect Aggregation and Chemical Degradation of Perovskite Solar Cells. J. Phys. Chem. Lett. 2018, 9, 2196-2201.

(60) Zhang, W. B.; Yu, N.; Yu, W. Y.; Tang, B. Y. Stability and Magnetism of Vacancy in NiO: A GGA+U Study. Eur. Phys. J. B 2008, 64, 153-158.

(61) Osorio-Guillén, J.; Lany, S.; Zunger, A. Nonstoichiometry and Hole Doping in NiO. AIP Conf. Proc. 2010, 1199, 128-129.

(62) Jang, W.-L.; Lu, Y.-M.; Hwang, W.-S.; Hsiung, T.-L.; Wang, H. P. Point Defects in Sputtered NiO Films. Appl. Phys. Lett. 2009, 94, 062103.

(63) Kojima, A.; Teshima, K.; Shirai, Y.; Miyasaka, T. Organometal Halide Perovskites as VisibleLight Sensitizers for Photovoltaic Cells. J. Am. Chem. Soc. 2009, 131, 6050-6051.

(64) Greiner, M. T.; Helander, M. G.; Wang, Z.-B.; Tang, W.-M.; Lu, Z.-H. Effects of Processing Conditions on the Work Function and Energy-Level Alignment of NiO Thin Films. J. Phys. Chem. C 2010, 114, 19777-19781.

(65) Madjid, A. H.; Martinez, J. M. Thermionic Emission from Nickel Oxide. Phys. Rev. Lett. 1972, 28, 1313-1315.

(66) Olivier, J.; Servet, B.; Vergnolle, M.; Mosca, M.; Garry, G. Stability/Instability of Conductivity and Work Function Changes of ITO Thin Films, UV-Irradiated in Air or Vacuum: Measurements by the Four-Probe Method and by Kelvin Force Microscopy. Synth. Met. 2001, 122, 87-89.

(67) Hu, L.; Peng, J.; Wang, W.; Xia, Z.; Yuan, J.; Lu, J.; Huang, X.; Ma, W.; Song, H.; Chen, W.; Cheng, Y.-B.; Tang, J. Sequential Deposition of $\mathrm{CH}_{3} \mathrm{NH}_{3} \mathrm{PbI}_{3}$ on Planar NiO Film for Efficient Planar Perovskite Solar Cells. ACS Photonics 2014, 1, 547-553.

(68) Zhai, Z.; Huang, X.; Xu, M.; Yuan, J.; Peng, J.; Ma, W. Greatly Reduced Processing Temperature for a Solution-Processed $\mathrm{NiO}_{x}$ Buffer Layer in Polymer Solar Cells. Adv. Energy Mater. 2013, 3, 1614-1622.

(69) Kamiya, T.; Ohta, H.; Kamiya, M.; Nomura, K.; Ueda, K.; Hirano, M.; Hosono, H. Li-Doped $\mathrm{NiO}$ Epitaxial Thin Film with Atomically Flat Surface. J. Mater. Res. 2004, 19, 913-920.

(70) Jang, W.-L.; Lu, Y.-M.; Hwang, W.-S.; Chen, W.-C. Electrical Properties of Li-Doped NiO Films. J. Eur. Ceram. Soc. 2010, 30, 503-508.

(71) Park, M.-A.; Park, I. J.; Park, S.; Kim, J.; Jo, W.; Son, H. J.; Kim, J. Y. Enhanced Electrical Properties of Li-Doped $\mathrm{NiO}_{x}$ Hole Extraction Layer in p-i-n Type Perovskite Solar Cells. Curr. Appl. Phys. 2018, 18, S55-S59. 


\section{Graphical TOC Entry}

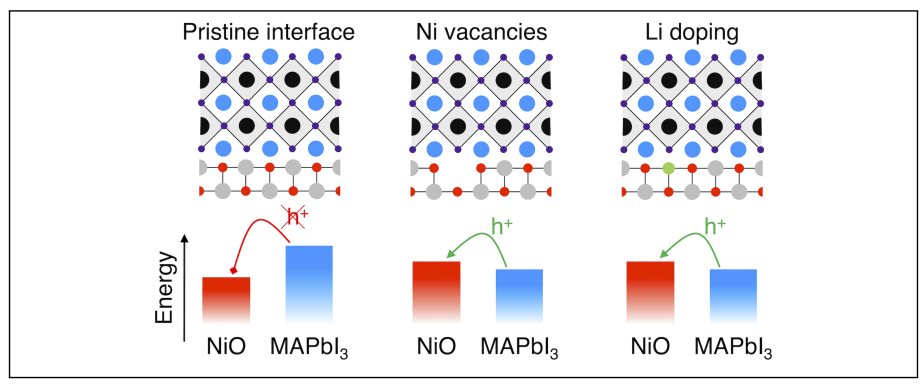

